\title{
Unexpected Sensitivity of Nitrogen Ions Superradiant Emission on Pump Laser Wavelength and Duration
}

\author{
Yi Liu, ${ }^{1,2}$ Pengji Ding, ${ }^{2,3}$ Neven Ibrakovic, ${ }^{3}$ Samuel Bengtsson, ${ }^{3}$ Shihua Chen, ${ }^{4}$ Rostyslav Danylo, ${ }^{1,2}$ \\ Emma R. Simpson, ${ }^{3}$ Esben W. Larsen, ${ }^{3}$ Xiang Zhang, ${ }^{1}$ Zhengquan Fan, ${ }^{1}$ Aurélien Houard, ${ }^{2}$ Johan Mauritsson, ${ }^{3}$ \\ Anne L'Huillier, ${ }^{3}$ Cord L. Arnold, ${ }^{3}$ Songlin Zhuang, ${ }^{1}$ Vladimir Tikhonchuk, ${ }^{5}$ and André Mysyrowicz ${ }^{2, *}$ \\ ${ }^{1}$ Shanghai Key Lab of Modern Optical System, University of Shanghai for Science and Technology, \\ 516, Jungong Road, 200093 Shanghai, China \\ ${ }^{2}$ Laboratoire d'Optique Appliquée, ENSTA ParisTech, CNRS, Ecole polytechnique, Université Paris-Saclay, \\ 828 Boulevard des Maréchaux, 91762 Palaiseau cedex, France \\ ${ }^{3}$ Department of Physics, Lund University, SE-22100 Lund, Sweden \\ ${ }^{4}$ Department of Physics, Southeast University, 211189, Nanjing, China \\ ${ }^{5}$ Centre Lasers Intenses et Applications, Université de Bordeaux, CEA, CNRS, Talence 33405, France
}

(Received 2 July 2017; published 17 November 2017)

\begin{abstract}
Nitrogen molecules in ambient air exposed to an intense near-infrared femtosecond laser pulse give rise to cavity-free superradiant emission at 391.4 and $427.8 \mathrm{~nm}$. An unexpected pulse duration-dependent cyclic variation of the superradiance intensity is observed when the central wavelength of the femtosecond pump laser pulse is finely tuned between 780 and $820 \mathrm{~nm}$, and no signal occurs at the resonant wavelength of $782.8 \mathrm{~nm}\left(2 \omega_{782.8 \mathrm{~nm}}=\omega_{391.4 \mathrm{~nm}}\right)$. On the basis of a semiclassical recollision model, we show that an interference of dipolar moments of excited ions created by electron recollisions explains this behavior.
\end{abstract}

DOI: 10.1103/PhysRevLett.119.203205

Cavity-free "lasing" from ionized nitrogen molecules is a fascinating and challenging problem. By focusing a linearly polarized femtosecond laser pulse at $800 \mathrm{~nm}$ in ambient air or pure nitrogen, one observes in the forward direction an intense directional and linearly polarized emission at 391.4 and/or $427.8 \mathrm{~nm}$ [1-6]; see Fig. 1(a). These two wavelengths correspond to transitions from the second excited state $B^{2} \Sigma_{u}^{+}$to the fundamental state $X^{2} \Sigma_{g}^{+}$of singly ionized nitrogen molecules with the vibrational quantum number $\nu=0$ or 1 [6]; see Fig. 1(b). Recent time-resolved measurements have shown that these emissions are in fact superradiant in nature $[7,8]$. Superradiance is a phenomenon that occurs when an ensemble of emitters interacts with a common light field in a collective and coherent fashion, leading to a characteristic short emission burst, which is drastically different from the exponentially decaying spontaneous emission produced by an ensemble of isolated emitters [7-9]. An energy enhancement by 2 orders of magnitude was observed when a short seed pulse around $391.4 \mathrm{~nm}$ was injected collinearly with the pump pulse inside the nitrogen gas $[8,10]$. Microjoules of energy were obtained at $427.8 \mathrm{~nm}$ by simply sending a femtosecond pulse with $200 \mathrm{~mJ}$ energy at $800 \mathrm{~nm}$ in air [3]. Since the emission has a duration of a few picoseconds and arises

Published by the American Physical Society under the terms of the Creative Commons Attribution 4.0 International license. Further distribution of this work must maintain attribution to the author(s) and the published article's title, journal citation, and DOI. from several filaments of diameter 50-100 $\mu \mathrm{m}[7,8]$, the local peak intensity of the $427.8 \mathrm{~nm}$ radiation can reach $\sim 10^{10} \mathrm{~W} / \mathrm{cm}^{2}$. Together with the fact that filament can be formed at kilometer distances from the laser source [11], this provides a promising tool for atmospheric remote sensing and diagnostics [12].

The physical mechanism underlying this optical gain is, however, controversially discussed in the literature $[2,6,8,13,14]$. One interpretation is based on the laserfield-induced nitrogen molecule alignment $[13,14]$. According to this model, a stimulated emission occurs as a result of a transient inversion between rotational distributions of excited level $B^{2} \Sigma_{u}^{+}$and the ground state
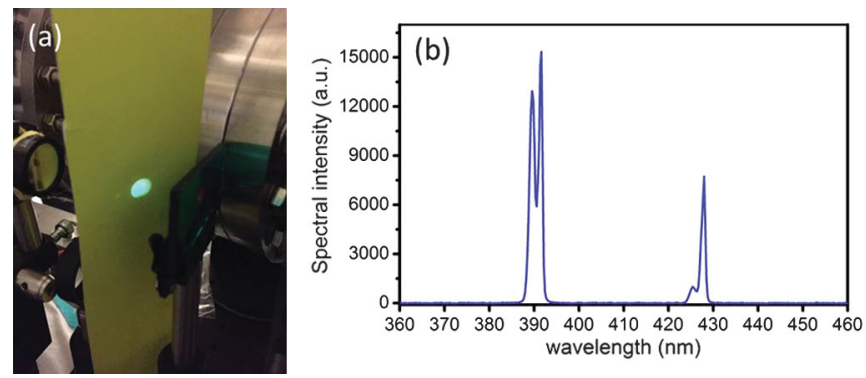

FIG. 1. (a) Forward $391.4 \mathrm{~nm}$ emission observed on a yellow fluorescent paper, after the pump laser around $800 \mathrm{~nm}$ was filtered out by the color glass filter (BG 40). (b) Experimental spectrum of the forward emission. The doublet structure corresponds to the $R$ and $P$ bands of the nitrogen ions. The experiments were performed with a nitrogen pressure of 5 mbar and a pump laser energy of $1.1 \mathrm{~mJ}$. 
$X^{2} \Sigma_{g}^{+}$. Another interpretation assumes the pump pulse initiates radiative transfers of the population from the ground state $X^{2} \Sigma_{g}^{+}$to two excited states of the molecular ion, $B^{2} \Sigma_{u}^{+}$and $A^{2} \Pi_{u}$. Depletion of the level $X^{2} \Sigma_{g}^{+}$by these two radiative processes leads to a steady state regime of population inversion between the levels $B^{2} \Sigma_{u}^{+}$and $X^{2} \Sigma_{g}^{+}$, because most of the nitrogen ion population is stored in the intermediate $A^{2} \Pi_{u}$ state $[2,6]$. Some of the authors of this Letter proposed that inelastic electron recollisions play an essential role in the superradiant process [8]. This latter interpretation is supported by the fact that the optical gain depends on the pump pulse polarization. It was observed that the lasing intensity decreased significantly when the pump laser ellipticity increases beyond 0.2 . This behavior, similar to that encountered in laser-driven high-order harmonic generation (HHG) [15,16], suggests the same semiclassical interpretation of electron recollision excitation. Only a linear or slightly elliptical laser field can force an ionized electron to return to the molecular ion with a significant kinetic energy. The resulting recollision is at the origin of many physical processes such as HHG [15,16], nonsequential double ionization [17], molecular fragmentation [18], formation of high-order Rydberg atoms [19], and inner-shell electronic excitation of molecules [20].

The purpose of this Letter is to further examine the recollision scenario. We experimentally measured the superradiant emission as a function of the wavelength and duration of the pump laser. A marked cyclic variation of the superradiant intensity is observed when the wavelength of the pump laser is varied over a small interval around $800 \mathrm{~nm}$, while the fluorescence is almost constant. The superradiance is also observed to depend sensitively upon the pump pulse duration. These results can be explained by the recollision excitation model. It is found that the observed cyclic variations of superradiant intensity are due to the interference between the dipole excitation probabilities from different electron trajectories. This leads us to conclude that the dipolar moment induced by the electron recollision plays an essential role in the superradiance of nitrogen ions.

In the experiments, we measured the superradiant intensity as a function of the pump pulse wavelength, using a tunable Ti:sapphire femtosecond laser system. This laser system includes two programable acousto-optical filters (DAZZLER and MAZZLER, Fastlite), which are used to increase and shape the gain bandwidth up to $100 \mathrm{~nm}$, supporting either the shortest pulses of $\sim 20 \mathrm{fs}$ or longer pulses with a reduced bandwidth and a central wavelength tunable between 780 and $820 \mathrm{~nm}$. In our experiments, the pulses were focused with a reflective focusing mirror into a gas chamber with nitrogen gas at 2-5 mbar pressure. The numerical aperture of the beam focusing was $\mathrm{NA}=0.025$. The forward emitted signal around $391.4 \mathrm{~nm}$ was detected by a fiber spectrometer
(Ocean Optics, HR4000) or a photodiode behind a narrow bandwidth $(10 \mathrm{~nm})$ interference filter centered at $390 \mathrm{~nm}$.

In a first experiment, the laser pulse bandwidth was reduced in order to achieve tunability of the central laser wavelength. The pulse was kept Fourier transform limited (without a frequency chirp), and its energy was kept at $0.9 \pm 0.05 \mathrm{~mJ}$. The experimental results are presented in Figs. 2(a)-2(c) for pulse durations of 35, 40, and 50 fs. We observe a cyclic variation of the superradiance with the pump pulse wavelength, and a minimum signal at the resonant wavelength $\left(2 \omega_{782.8 \mathrm{~nm}}=\omega_{391.4 \mathrm{~nm}}\right)$ around $782.8 \mathrm{~nm}$ independent of the laser pulse duration. In addition, the period of the cycles decreases for a longer

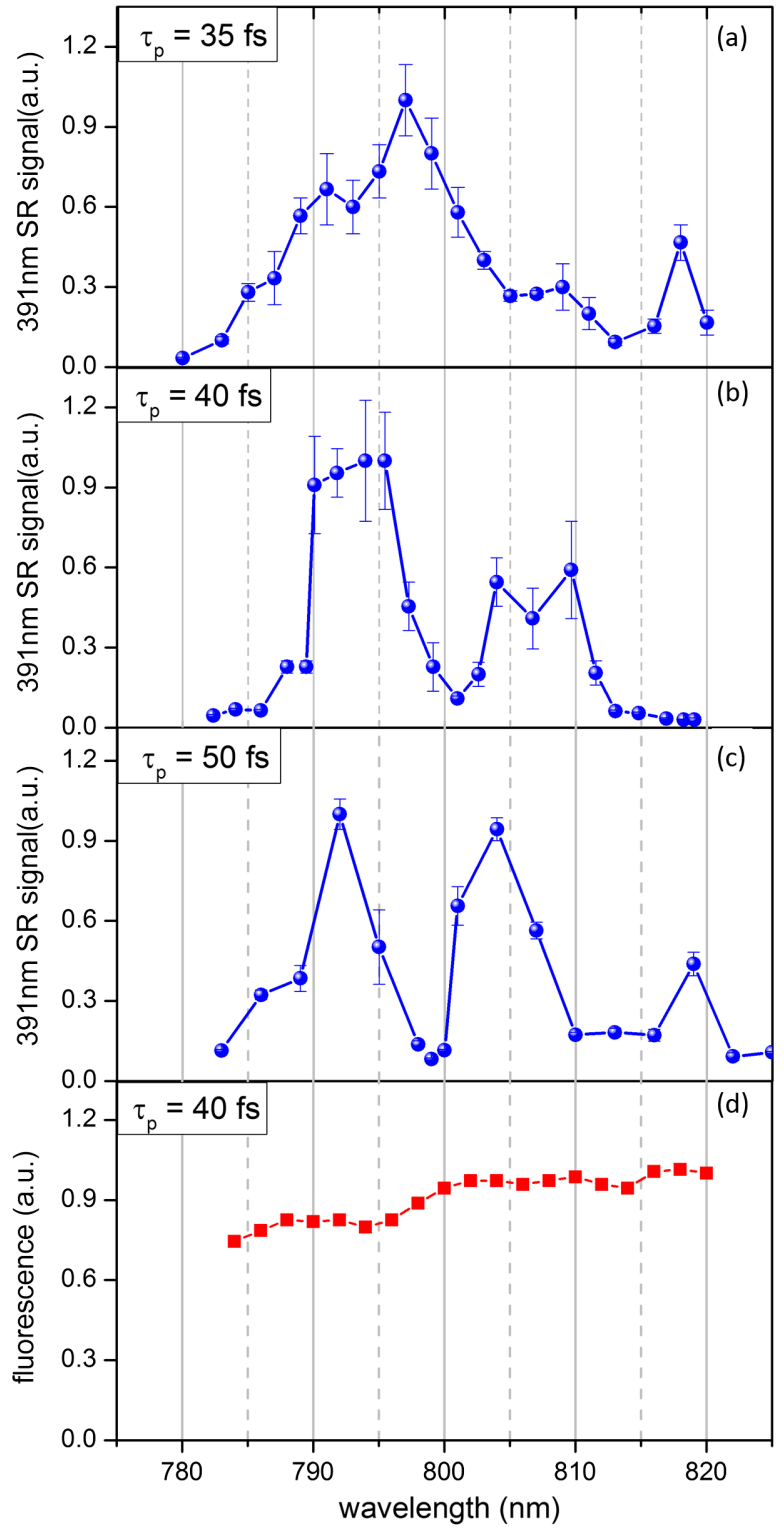

FIG. 2. (a)-(c) Measured forward $391.4 \mathrm{~nm}$ superradiance signal as a function of the pump laser central wavelength for pulse durations of 35, 40, and $50 \mathrm{fs}$. (d) Sideways fluorescence at $391.4 \mathrm{~nm}$ in the case of a $40 \mathrm{fs}$ incident pump laser pulse. The gas pressure in (b) was 2.5 and 5 mbar for (a), (c), and (d). 
pulse duration. This unexpected cyclic behavior of the emission signal is in clear contrast to the number of ions in the excited state, which is measured by detecting the sideway fluorescence at the wavelength of $391.4 \mathrm{~nm}$ as a function of the laser wavelength. Figure 2(d) shows that the fluorescence is practically independent of the laser wavelength, except a slight increase which can be due to laser beam alignment drift during measurement. That is, the creation of a population of excited ions is necessary but not sufficient for producing the superradiant emission.

In order to shine further light on the emission mechanism, we conducted a second series of experiments, where the pulse duration was changed while the laser central wavelength was kept at $800 \mathrm{~nm}$. The pulse peak power was equal to $16 \mathrm{GW}$, and it was always kept Fourier transform limited. By increasing the pulse duration from 21 to $46 \mathrm{fs}$, we observed that the emission signal first increases to a maximum for a pulse duration of $26 \mathrm{fs}$ and then decreases to an undetectable level for a longer pulse duration (Fig. 3). As will be discussed below, these results can be explained by the recollision excitation model and therefore support the interpretation of recollisions as being at the heart of the superradiance.

Let us consider a nitrogen molecule $\mathrm{N}_{2}$ with ionization potential $U_{i}$ in a linearly polarized laser field with central frequency $\omega$ and instantaneous electric field $E(t)=E_{0}(t)$ $\cos \omega t$. The ionization process at a laser intensity on the order of $I=1.5 \times 10^{14} \mathrm{~W} / \mathrm{cm}^{2}$ corresponds to a Keldysh parameter on the order of 1 , and the ionization rate is given by the time-dependent Perelomov-Popov-Terent'ev (PPT) theory [21]. An electron liberated from the nitrogen molecule at time $t_{0}$ follows a deterministic trajectory governed by the classical equations of motion. There is a finite probability that the electron returns to the parent ion with a kinetic energy sufficient for promoting the ion to its

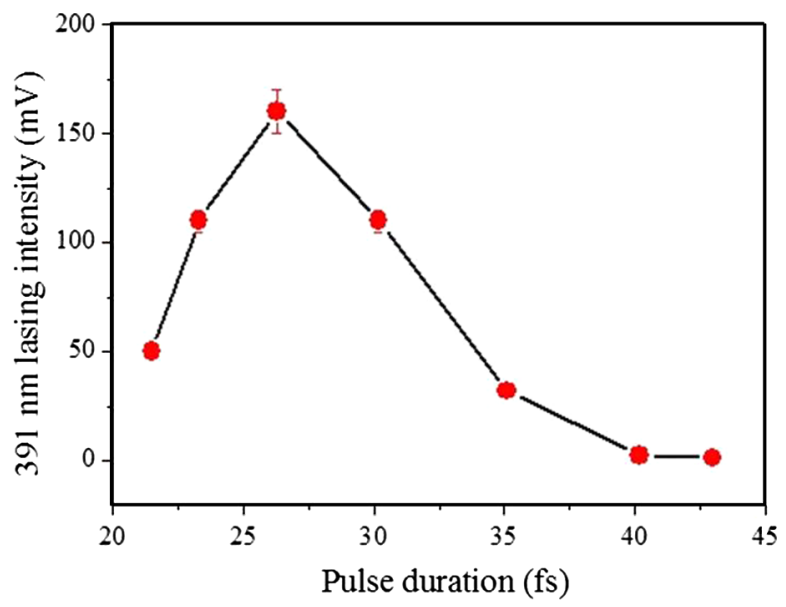

FIG. 3. Experimentally measured $391.4 \mathrm{~nm}$ lasing signal for different pulse durations with a constant central wavelength at $800 \mathrm{~nm}$. The nitrogen gas pressure was 5 mbar. The dots present the experimental measurements. excited state $B^{2} \Sigma_{u}^{+}$. In view of the correlated electron motion in the laser field, a description of the ion excitation process in terms of rate equations describing the evolution of populations is inadequate, and it is also necessary to consider the dipolar moment formation.

In our model, we solve the classical one-dimensional (1D) equations for the electron position $x_{e}$ and the velocity $v_{e}$ in the laser field $E(t)$ polarized along the $x$ direction:

$$
\frac{d v_{e}}{d t}=-\frac{e}{m_{e}} E(t), \quad \frac{d x_{e}}{d t}=v_{e},
$$

assuming that at the starting time $t_{0}$ each free electron satisfies the conditions $v_{e}\left(t_{0}\right)=0$ and $x_{e}\left(t_{0}\right)=0$ simultaneously [16]. The probability of excitation of the parent ion in the recollision process has been calculated as follows:

$$
w_{B X}\left(t_{0}\right)=w_{\text {ion }}\left(t_{0}\right) w_{\text {rec }}\left(t_{0}\right) w_{\text {exc }}\left(t_{s}\right),
$$

where $w_{\text {ion }}\left(t_{0}\right)$ is the ionization probability given by the PPT theory [21] and $w_{\text {rec }}\left(t_{0}\right)$ is the recollision probability, which in our simplified 1D model (1) equals 1 if the electron returns to the ion or 0 elsewhere. Finally, $w_{\mathrm{exc}}\left(t_{s}\right)$ is the ion excitation probability in the recollision. It is equal to 0 , if the kinetic energy of returning electron at the moment of recollision $t_{s}$ is smaller than the energy of transition from the $X^{2} \Sigma_{g}^{+}$to $B^{2} \Sigma_{u}^{+}$state, $\varepsilon_{e}\left(t_{s}\right)<\hbar \omega_{B X}=$ $3.17 \mathrm{eV}$. Conversely, $w_{\mathrm{exc}}\left(t_{s}\right)=V_{0}^{2} \cong 0.01$, if the energy of the recolliding electron is larger than the ion excitation energy [8]. Here $V_{0}^{2}$ can be considered as the probability of ion excitation in a single collision with an electron. Compared to our previous model [8], we consider here only the first recollision event of each electron because of the quantum dispersion of the electron wave function. The total probability of excitation $W_{B X}$ is obtained by a superposition of all excitation events of electrons born at different times:

$$
\mathrm{W}_{B X}=\int_{-\infty}^{+\infty} w_{B X}\left(t_{0}\right) d t_{0} .
$$

We have calculated the dependence of the excitation probability as a function of the pump laser wavelength over the frequency interval $780-820 \mathrm{~nm}$. The calculated ion excitation probability is approximately $3.5 \%-3.7 \%$ in the considered range of parameters. In particular, it decreases as a function of the pump laser wavelength by less than $10 \%$ over the frequency interval $780-820 \mathrm{~nm}$. This fact is in agreement with our experimental observation that the side fluorescence from the plasma remained unchanged when varying the pump laser wavelength, since it is proportional to the number of excited ions in the state $B^{2} \Sigma_{u}^{+}$[see Fig. 2(d)].

Let us now turn to the dipole moment, which can serve as a trigger to launch the superradiance process. While the 
(a)

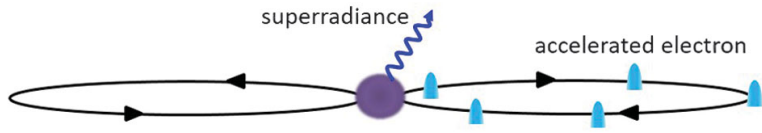

(b)

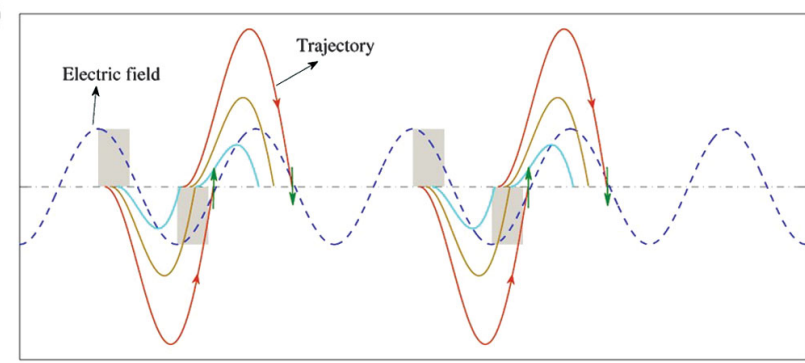

FIG. 4. (a) Schematic illustration of electron recollision excitation. (b) Trajectories of the electrons (solid lines) in the laser field (blue dashed line), which lead to the periodic recollision excitation of the parent ion. The recollision occurs from opposite sides of the parent ions, leading to interference of the dipole moments, with the direction of the recolliding electrons indicated by the arrow (green).

absolute value of the ion dipole moment is known, $d_{B X} \approx$ 0.7 a.u. [22], its direction and phase depend on the interaction conditions, shown schematically in Fig. 4. The dipole moment is calculated according to the following expression: $p_{B X}\left(t_{0}\right)=w_{B X}\left(t_{0}\right) \sigma\left(t_{s}\right) \exp \left(-i \omega_{B X} t_{s}\right)$, where $\sigma\left(t_{s}\right)= \pm 1$ accounts for the direction that the electron approaches the parent ion with respect to the $x$ axis and the last factor accounts for the dipole phase at the moment of recollision. Similarly to the excitation probability $W_{B X}$, the dipolar moment probability $P_{B X}$ can be represented as an integral over the laser pulse duration:

$$
P_{B X}=\int_{-\infty}^{+\infty} w_{B X}\left(t_{0}\right) \sigma\left(t_{s}\right) \exp \left(-i \omega_{B X} t_{s}\right) d t_{0}
$$

We have calculated expression (4) numerically, assuming a laser pulse of constant amplitude with an intensity of $I=1.5 \times 10^{14} \mathrm{~W} / \mathrm{cm}^{2}$, in order to simplify the calculations and the analysis presented later. The predicted dependence of the dipole moment as a function of detuning is shown in Fig. 5(a) for the pump pulse durations $\tau_{p}=35$, 40, and $50 \mathrm{fs}$. Three features are noticeable. First, the model predicts a zero dipole moment regardless of the pulse duration if the laser is tuned to the wavelength of $782.8 \mathrm{~nm}$, where the second harmonic of the laser matches the $391.4 \mathrm{~nm}$ transition frequency $\left(2 \omega_{782.8 \mathrm{~nm}}=\omega_{391.4 \mathrm{~nm}}\right)$. Second, a cyclic variation of the dipole moment is found for a very fine tuning of the central wavelength with a slightly decreasing amplitude for a longer wavelength. Finally, the period of the cycles decreases for longer laser pulses. We also calculated the dipole moment for a laser pulse at a central wavelength of $800 \mathrm{~nm}$ with different pulse durations. The result is presented in Fig. 5(b). An optimal pulse duration around $30 \mathrm{fs}$ is predicted, while either
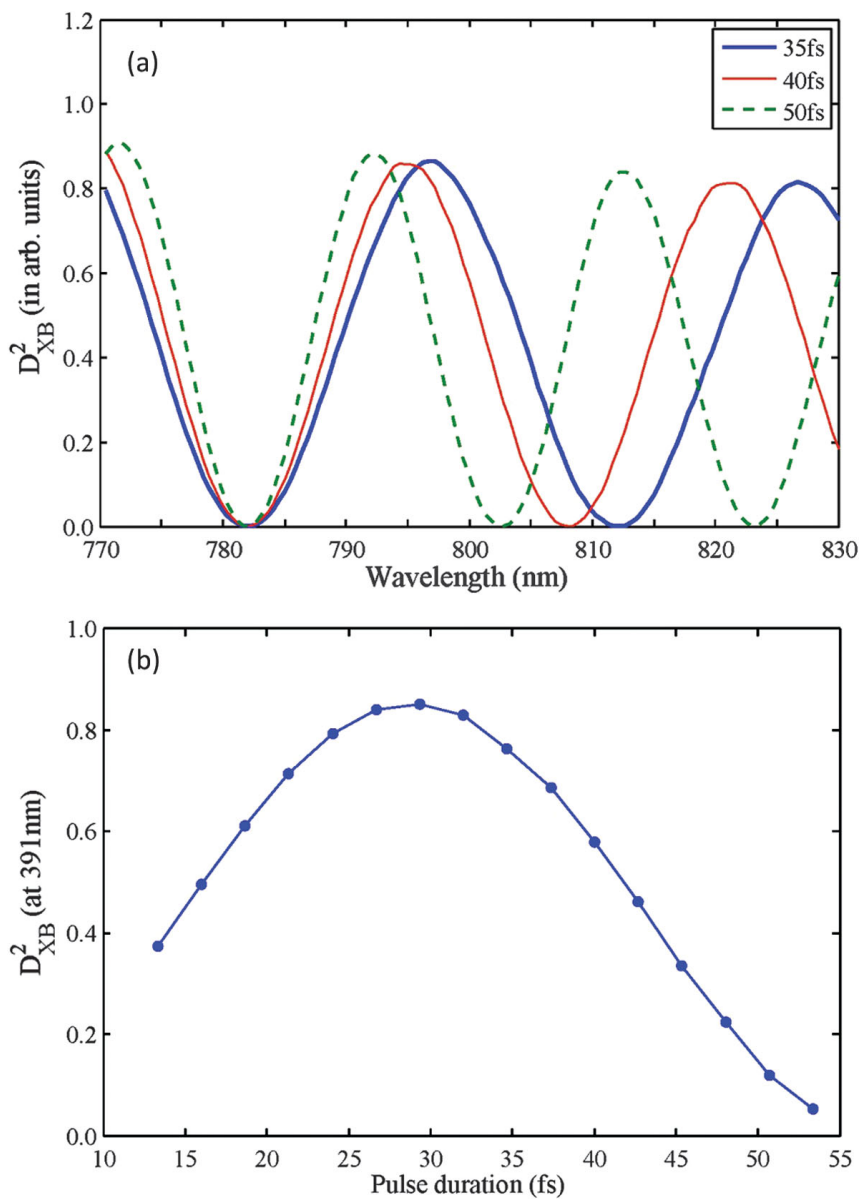

FIG. 5. Calculated average dipolar momentum of the state after the laser-molecule interaction: (a) as a function of the laser wavelength for the pulse durations 35, 40, and $50 \mathrm{fs}$; (b) as a function of the pulse duration for the laser wavelength of $800 \mathrm{~nm}$.

shorter or longer pulses lead to a smaller dipole moment. All simulations of the dipole moment from the recollision model agree well with the experimental results.

How should we understand the above simulation results? The dipole moment in Eq. (4) can be written as a sum over the contributions from all laser periods. Because of the periodicity of the functions entering in Eq. (4), the only additional factor is $\exp \left[-2 \pi i m \omega_{B X} / \omega\right]$, where $m$ is the period number. It accounts for the difference between the phase of the dipole oscillations and the phase of the laser field. Moreover, the contributions from subsequent halfcycles enter with opposite signs as the dipole moment is represented by an impair function $\sigma\left(t_{s}\right)$, as depicted in Fig. 4(b). This brings an additional factor $\exp \left[-i \pi \omega_{B X} / \omega\right]$ in each odd half-cycle. Then Eq. (4) reads

$$
\begin{aligned}
P_{B X}= & \sum_{m=1}^{M} \int_{0}^{\pi / \omega} w_{B X}\left(t_{0}\right) \exp \left[-i \omega_{B X}\left(t_{s}+2 \pi m / \omega\right)\right] d t_{0} \\
& \times\left[1-\exp \left(-i \pi \omega_{B X} / \omega\right)\right],
\end{aligned}
$$


where the integral is taken over half a laser period for a pulse of $M$ periods. It can be further simplified by noting that the last factor in square brackets is independent on the period number:

$$
\begin{aligned}
P_{B X}= & 2 i \sin \left(\pi \omega_{B X} / 2 \omega\right) \sum_{m=1}^{M} \int_{0}^{\pi / \omega} w_{B X}\left(t_{0}\right) \\
& \times \exp \left(-i \omega_{B X} t_{s}\right) d t_{0} \exp \left[-i \pi(2 m+1 / 2) \omega_{B X} / \omega\right] .
\end{aligned}
$$

This expression shows a particular property of the transition from $B^{2} \Sigma_{u}^{+}$to $X^{2} \Sigma_{g}^{+}$states: The dipole moment is zero if the transition frequency is twice the driver frequency $\omega_{B X}=2 \omega$. This parity selection is due to the symmetry of the ion electronic states, similarly to the case of HHG where only odd harmonics are allowed. As the frequency of the laser is tuned away from the resonance, a phase shift develops between each positive cycle and its successive negative cycles, preventing this total cancellation. However, with further frequency detuning, the interference between successive laser periods can eventually lead to a secondary minimum. To evaluate the corresponding frequency detuning, we note that the sum over laser periods in Eq. (5) presents a geometrical progression and can be performed analytically (we removed the phase factors which are not important for the further analysis):

$P_{B X} \cong \frac{\sin \left(\pi M \omega_{B X} / \omega\right)}{\cos \left(\pi \omega_{B X} / 2 \omega\right)} \int_{0}^{\pi / \omega} \exp \left(-i \omega_{B X} t_{s}\right) w_{B X}\left(t_{0}\right) d t_{0}$.

The term in the numerator accounts for the interference in the polarization excitation. Destructive interference occurs when the numerator goes through zero, i.e., $M \omega_{B X}=n \omega$, where $n$ is an integer. The main resonance condition corresponds to $n=2 M$, while additional zeros occur for $n=2 M \pm k$, where $k$ is an integer. Then, replacing $M$ with the laser pulse duration $\tau_{p}=2 \pi M / \omega$, we find the following condition for the destructive interference: $\omega_{d}=\omega_{B X} / 2 \pm k \pi / \tau_{p}$. That is, the first additional minimum corresponds to the frequency detuning $\Delta \omega_{d}= \pm \pi / \tau_{p}$, which is inversely proportional to the pulse duration. For a pulse of 13 cycles ( $\left.\tau_{p}=35 \mathrm{fs}\right)$, a minimum is expected for the detuning $\Delta \omega_{d}=0.09 \mathrm{fs}^{-1}$, or $\Delta \lambda_{d}=30 \mathrm{~nm}$, which agrees well with the simulation result shown in Fig. 5(a). Conversely, for a given laser wavelength, the optimum amplification condition $\Delta \omega_{m}=$ $\pm k \pi / 2 \tau_{p}$ depends on the laser pulse duration. For the laser wavelength of $800 \mathrm{~nm}$, the optimum pulse duration is $30 \mathrm{fs}$, as is shown in Fig. 5(b), and the dipole moment decreases with shorter and longer laser pulses.

In conclusion, a subtle cyclic dependence of the superradiance around $391.4 \mathrm{~nm}$ of singly ionized nitrogen molecular ions is observed upon a fine-tuning of the pump laser wavelength, with a period dependent on the duration of the pump laser. In addition, the emission minimum occurs at the laser central wavelength of $782.8 \mathrm{~nm}$, irrespective of the pulse duration. A semiclassical model based on the ion recollision excitation mechanism explains these three experimental features. It is shown that the superradiance follows the variations of the ion dipole moment induced by recollisions. This study shows that the optical gain of the nitrogen ions depends not only on the presence of the excited ions, but also essentially on their dipole moment. Thus, an interpretation of optical gain based only on a population inversion is not adequate for the considered transition.

The work is supported in part by the National Natural Science Foundation of China (Grants No. 11574213, No. 11174050, and No. 11474051), the European Research Council (PALP), the Knut and Alice Wallenberg Foundation, the Swedish Research Council, the Swedish Foundation for Strategic Research, the Crafoord Foundation, Innovation Program of Shanghai Municipal Education Commission (Grant No. 2017-01-07-00-07-E00007), Shanghai Science and Technology Commission (Grant No. 17060502500), and LASERLAB-EUROPE III [Grant Agreement No. 284464 (EU FP7)]. Y. L. acknowledges the support by The Program for Professor of Special Appointment (Eastern Scholar) at Shanghai Institutions of Higher Learning (No. TP2014046). The authors acknowledge stimulating discussion with Professor Hongbing Jiang and Chengyin Wu of Peking University and Dr. Luqi Yuan of Stanford University.

* Corresponding author. andre.mysyrowicz@ensta-paristech.fr

[1] W. Chu, G. Li, H. Xie, J. Ni, J. Yao, B. Zeng, H. Zhang, C. Jing, H. Xu, Y. Cheng, and Z. Xu, A self- induced white light seeding laser in a femtosecond laser filament, Laser Phys. Lett. 11, 015301 (2014).

[2] H. Xu, E. Lötstedt, A. Iwasaki, and K. Yamanouchi, Sub10-fs population inversion in $\mathrm{N}_{2}^{+}$in air lasing through multiple state coupling, Nat. Commun. 6, 8347 (2015).

[3] G. Point, Y. Liu, Y. Brelet, S. Mitryukovskiy, P. Ding, A. Houard, and A. Mysyrowicz, Lasing of ambient air with microjoule pulse energy pumped by a multi-terawatt femtosecond laser, Opt. Lett. 39, 1725 (2014).

[4] Y. Liu, Y. Brelet, G. Point, A. Houard, and A. Mysyrowicz, Self-seeded lasing action of air pumped by $800 \mathrm{~nm}$ femtosecond laser pulses, Opt. Express 21, 22791 (2013).

[5] T. Wang, J. Ju, J. F. Daigle, S. Yuan, R. Li, and S. L. Chin, Self-seeded forward lasing action from a femtosecond Ti: sapphire laser filament in air, Laser Phys. Lett. 10, 125401 (2013).

[6] J. Yao, S. Jiang, W. Chu, B. Zeng, C. Wu, R. Lu, Z. Li, H. Xie, G. Li, C. Yu, Z. Wang, H. Jiang, Q. Gong, and Y. Cheng, Population Redistribution among Multiple Electronic States of Molecular Nitrogen Ions in Strong Laser Fields, Phys. Rev. Lett. 116, 143007 (2016).

[7] G. Li, C. Jing, B. Zeng, H. Xie, J. Yao, W. Chu, J. Ni, H. Zhang, H. Xu, Y. Cheng, and Z. Xu, Signature of superradiance from a nitrogen-gas plasma channel produced 
by strong-field ionization, Phys. Rev. A 89, 033833 (2014).

[8] Y. Liu, P. Ding, G. Lambert, A. Houard, V. Tikhonchuk, and A. Mysyrowicz, Recollision-Induced Superradiance of Ionized Nitrogen Molecules, Phys. Rev. Lett. 115, 133203 (2015).

[9] M. Gross and S. Haroche, Superradiance: An essay on the theory of collective spontaneous emission, Phys. Rep. 93, 301 (1982).

[10] Y. Yao, G. Li, C. Jing, B. Zeng, W. Chu, J. Ni, H. Zhang, H. Xie, C. Zhang, H. Li, H. Xu, S. L. Chin, Y. Cheng, and $\mathrm{Z}$. $\mathrm{Xu}$, Remote creation of coherent emissions in air with two-color ultrafast laser pulses, New J. Phys. 15, 023046 (2013).

[11] M. Durand, A. Houard, B. Prade, A. Mysyrowicz, A. Durécu, B. Moreau, D. Fleury, O. Vasseur, H. Borchert, K. Diener, R. Schmitt, F. Théberge, M. Chateauneuf, J. F. Daigle, and J. Dubois, Kilometer range filamentation, Opt. Express 21, 26836 (2013).

[12] J. Ni, W. Chu, H. Zhang, B. Zeng, J. Yao, L. Qiao, G. Li, C. Jing, H. Xie, H. Xu, Y. Cheng, and Z. Xu, Impulsive rotational Raman scattering of $\mathrm{N}_{2}$ by a remote "air laser" in femtosecond laser filament, Opt. Lett. 39, 2250 (2014).

[13] A. Azarm, P. Corkum, and P. Polynkin, in Proceedings of CLEO: 2016 (Optical Society of America, Washington, DC, 2016), paper JTh4B.9.

[14] D. Kartashov, S. Haessler, S. Ališauskas, G. Andriukaitis, A. Pugžlys, A. Baltuška, J. Möhring, D. Starukhin, M. Motzkus, A. M. Zheltikov, M. Richter, F. Morales, O. Smirnova, M. Yu. Ivanov, and M. Spanner, in Research in Optical Sciences (Optical Society of America, Washington, DC, 2016), paper HTh4B.5.

[15] P. Dietrich, N. H. Burnett, M. Ivanov, and P. B. Corkum, High-harmonic generation and correlated two-electron multiphoton ionization with elliptically polarized light, Phys. Rev. A 50, R3585 (1994).

[16] J. L. Krause, K. J. Schafer, and K. C. Kulander, High-Order Harmonic Generation from Atoms and Ions in the High Intensity Regime, Phys. Rev. Lett. 68, 3535 (1992).

[17] V. R. Bhardwaj, D. M. Rayner, D. M. Villeneuve, and P. B. Corkum, Quantum Interference in Double Ionization and Fragmentation of $\mathrm{C}_{6} \mathrm{H}_{6}$ in Intense Laser Fields, Phys. Rev. Lett. 87, 253003 (2001).

[18] V. R. Bhardwaj, P. B. Corkum, and D. M. Rayner, Recollision during the High Laser Intensity Ionization of $\mathrm{C}_{60}$, Phys. Rev. Lett. 93, 043001 (2004).

[19] A. S. Landsman, A. N. Pfeiffer, C. Hofmann, M. Smolarski, C. Cirelli, and U. Keller, Rydberg state creation by tunnel ionization, New J. Phys. 15, 013001 (2013).

[20] Y. Deng, Z. Zeng, Z. Jia, P. Komm, Y. Zheng, X. Ge, R. Li, and G. Marcus, Ultrafast Excitation of an Inner-Shell Electron by Laser-Induced Electron Recollision, Phys. Rev. Lett. 116, 073901 (2016).

[21] A. M. Perelomov, V. S. Popov, and M. V. Terent'ev, Ionization of atoms in an alternating electric field, Sov. Phys. JETP 23, 924 (1966); V.S. Popov, Tunnel and multiphoton ionization of atoms and ions in a strong laser field (Keldysh theory), Phys. Usp. 47, 855 (2004).

[22] S. R. Langhoff and Ch. W. Bauschglicher, Jr., Theoretical study of the first and second negative systems of $\mathrm{N}_{2}^{+}, \mathrm{J}$. Chem. Phys. 88, 329 (1988). 